\title{
DESENVOLVIMENTO PROFISSIONAL, BURNOUT E CIDADANIA ORGANIZACIONAL
}

\author{
PROFESSIONAL DEVELOPMENT, BURNOUT AND ORGANIZATIONAL CITIZENSHIP
}

HARRISON BACHION CERIBELI

Professor Adjunto da Universidade Federal de Ouro Preto (UFOP) Doutor em Administração pela Universidade de São Paulo (FEARP/USP)

Orcid: http://orcid.org/0000-0002-1654-6273 / E-mail: harrisonbceribeli@ufop.edu.br Rua do Catete, no 166, Centro - Mariana/MG, CEP: 35420-057

\section{JOICE RAYRA VIEIRA DINIZ}

Universidade Federal de Ouro Preto (UFOP)

Graduada em Administração pela Universidade Federal de Ouro Preto (UFOP) Orcid: https://orcid.org/0000-0003-4641-157X / E-mail: joicervd@gmail.com

\section{RAONI DE OLIVEIRA INÁCIO}

Professor Adjunto da Universidade Federal de Ouro Preto (UFOP) Doutor em Administração pela Fundação Getúlio Vargas (EAESP/FGV) Orcid: http://orcid.org/0000-0003-3351-1061 / E-mail: raoni.inacio@ufop.edu.b

\section{ISRAEL JOSÉ DOS SANTOS FELIPE}

Professor Adjunto da Universidade Federal de Ouro Preto (UFOP) Doutor em Administração pela Fundação Getúlio Vargas (EAESP/FGV) Orcid: http://orcid.org/0000-0001-8608-0029 / E-mail: israelfelipe@ufop.edu.br

Submissão: 30/06/2020. Revisão: 19/04/2021. Aceite: 27/04/2021. Publicação: 28/07/2021. DOI: http://dx.doi.org/10.22277/rgo.v14i3.5619

\section{RESUMO}

Esta pesquisa objetivou analisar, no contexto da indústria cervejeira, a relação entre a síndrome de Burnout, as oportunidades de desenvolvimento profissional proporcionadas pelas organizações a seus funcionários e o comportamento de cidadania organizacional. Como método de pesquisa, empregou-se a survey, coletando-se dados junto a uma amostra composta por 384 funcionários de cervejarias multinacionais. Para análise, utilizou-se a técnica de Modelagem de Equações Estruturais. Analisando os resultados, constatou-se que, ao fornecer condições para que os funcionários das indústrias cervejeiras se desenvolvam, eles tendem a retribuir comportando-se como cidadãos organizacionais, além de estarem menos sujeitos a desenvolver a síndrome de Burnout. Complementarmente, confirmou-se que, entre os participantes da pesquisa, a síndrome de Burnout leva à redução da cooperação espontânea e da disposição de defender a imagem da organização.

Palavras-chave: Comportamento de cidadania organizacional. Desenvolvimento humano nas organizações. Esgotamento profissional. Cervejarias multinacionais.

Este é um artigo publicado em acesso aberto (Open Access) sob a licença Creative Commons Attribution, que permite uso, distribuição e reprodução em qualquer meio, sem restrições desde que o trabalho original seja corretamente citado. 


\begin{abstract}
This research aimed to analyze, in the context of the brewing industry, the relationship between Burnout syndrome, the professional development opportunities that are provided by the organizations to their employees and the organizational citizenship behavior. Survey was used as a research method, collecting data from a sample of 384 employees of multinational breweries. For analysis, Structural Equation Modeling technique was adopted. From the results, it was found that, by providing conditions for employees of the brewing industries to develop themselves, they tend to reciprocate by behaving as organizational citizens, as well as being less likely to develop Burnout syndrome. Complementarily, it was confirmed that, among the research participants, Burnout syndrome leads to a reduction in spontaneous cooperation and in the willingness to defend the organization's image.
\end{abstract}

Keywords: Organizational citizenship behavior. Human development in organizations. Professional exhaustion. Multinational breweries.

\title{
1 INTRODUÇÃO
}

O termo comportamento de cidadania organizacional (CCO) foi cunhado no início da década de 1980 pelos pesquisadores Smith, Organ, Near e Bateman (SMITH; ORGAN; NEAR, 1983; BATEMAN; ORGAN, 1983) para descrever uma postura de cooperação voluntária dos trabalhadores que desempenhavam, de maneira espontânea, tarefas que ultrapassavam as responsabilidades dos cargos para os quais haviam sido contratados.

Subsequentemente, à medida que diferentes benefícios provenientes desse tipo de conduta dos funcionários foram descortinados, gestores e estudiosos atribuíram cada vez maior importância à cidadania organizacional (FERDUS; KABIR, 2018), que passou a ser vista como elemento fundamental no cenário contemporâneo, no qual o capital humano assumiu um relevante papel no desenvolvimento de novos diferenciais competitivos para os negócios e implantação das estratégias organizacionais (MARTINSA; COSTA; SIQUEIRA, 2016; SOARES; SIENA, 2018). Com isso, o número de pesquisas voltadas à análise dos antecedentes do CCO aumentou consideravelmente (PODSAKOFF et al., 2000; READER et al., 2017).

Depois de realizar um levantamento nas bases Scopus, Scielo, Redalyc e Spell, combinando de diferentes maneiras os termos (em inglês e também português) "organizational citizenship", "human development", "employee development", "Burnout" e "employee exhaustion", limitando a busca, especificamente no Scopus, ao título do artigo, resumo e palavras-chave, e à área de "Business, Management and Accounting", identificouse a escassez de estudos correlacionando o CCO a duas temáticas que tem sido alvo de crescente interesse na atualidade: Burnout e o desenvolvimento humano nas organizações. Diante disso, definiu-se como objetivo de pesquisa analisar, no contexto das indústrias cervejeiras, a relação entre a síndrome de Burnout, as oportunidades de desenvolvimento profissional que são proporcionadas pelas organizações a seus funcionários e o comportamento de cidadania organizacional.

Optou-se por realizar esta pesquisa junto a profissionais que atuam na indústria cervejeira, a qual, além de movimentar o agronegócio nacional, dado que utiliza matériasprimas como malte, lúpulo e cevada, também impacta positivamente diversos outros setores econômicos, tais como o de transporte, energia, alumínio e vidro, sendo responsável pela geração de milhares de empregos no Brasil e pela movimentação de bilhões de reais na 
Harrison Bachion Ceribeli, Joice Rayra Vieira Diniz, Raoni de Oliveira Inácio e Israel José dos Santos Felipe

economia brasileira, o que se deve ao fato de que o país é o terceiro maior produtor mundial de cerveja (SINDICERV, 2019).

Esse setor, apesar de ser caracterizado como um oligopólio dominado pela primeira multinacional do Brasil, a Anheuser-Busch InBev ou simplesmente AB InBev (GONTIJO; GESSNER, 2015), é muito dinâmico em decorrência da falta de barreiras à entrada de novos competidores e da ampla possibilidade de diferenciação da oferta, o que faz com que as cervejarias busquem continuamente criar conhecimentos, aperfeiçoar os produtos e inovar (LIMBERGER; MARTINS, 2018), sendo necessário, para isso, investir em seu capital humano e aproveitá-lo como fonte de vantagem competitiva.

A justificativa para realização deste trabalho reside na possibilidade que se vislumbrou de contribuir para a compreensão dos antecedentes do CCO, auxiliando os gestores em seus esforços voltados para estimular os funcionários a se comportarem como verdadeiros cidadãos organizacionais, o que tende a aumentar a produtividade individual, levando a melhorias tanto no desempenho das equipes de trabalho quanto no desempenho das organizações (MARTINSA; COSTA; SIQUEIRA, 2016). Compete acrescentar que a relação entre os construtos aqui abordados carece de estudos, tanto na literatura nacional quanto na internacional, para ser adequadamente compreendida, o que evidencia o potencial que esta investigação apresenta de colaborar para a construção de um arcabouço teórico consistente acerca do comportamento de cidadania organizacional e seus antecedentes.

\section{REFERENCIAL TEÓRICO}

\subsection{COMPORTAMENTO DE CIDADANIA ORGANIZACIONAL}

O comportamento de cidadania organizacional (CCO) pode ser definido como uma postura voluntária do funcionário, que decorre de uma escolha pessoal, de executar tarefas não usuais e não contratuais (SMITH; ORGAN; NEAR, 1983) com o intuito de contribuir para o bom andamento das atividades da organização (MIAO; HUMPHREY; QIAN, 2017) e a melhoria dos resultados do negócio (SOARES; SIENA, 2018). O CCO possui sete dimensões: altruísmo, conscienciosidade, esportividade, lealdade organizacional, iniciativa individual, autodesenvolvimento e virtude cívica (REGO, 2002; PORTO; TAMAYO, 2005).

$\mathrm{O}$ altruísmo refere-se à disposição do indivíduo de colaborar com os demais (SMITH; ORGAN; NEAR, 1983), encorajando-Os, auxiliando-os em seu desenvolvimento pessoal (WILLIAMS; ANDERSON, 1991) e apresentando uma preocupação desinteressada com relação aos trabalhos em atraso ou de difícil execução (LEAL et al., 2009).

Por sua vez, a conscienciosidade remete ao consentimento e internalização das regras, regulamentos e procedimentos organizacionais (SMITH; ORGAN; NEAR, 1983), levando à execução minuciosa do trabalho (REGO, 2002).

A esportividade está relacionada a uma postura positiva e à tolerância do trabalhador ante comportamentos inoportunos por parte dos colegas (PODSAKOFF et al., 2000), assim como à transigência em relação às inclinações de cada indivíduo com quem convive no âmbito de seu trabalho (ORGAN, 1983).

Já a lealdade organizacional refere-se ao compromisso do sujeito de proteger a imagem da organização diante de possíveis distorções ou ameaças externas (CORDEIRO; CUNHA, 2018), promovendo uma representação conveniente de seu empregador (BETTENCOURT; GWINNER; MEUTER, 2001) e permanecendo dedicado às suas funções mesmo em condições desfavoráveis (MENEZES; AGUIAR; BASTOS, 2016). 
A iniciativa individual engloba a adoção, por parte dos funcionários, de ações facultativas (GEORGE; JONES, 1997) e medidas inovadoras para sanar os problemas e facilitar os processos de trabalho (GRAHAM, 1991), melhorando o desempenho organizacional (MOTOWIDLO; BORMAN; SCHMIT, 1997). Ademais, inclui a disposição do profissional para aceitar desafios (PODSAKOFF et al., 2000).

$O$ autodesenvolvimento relaciona-se à busca proativa do trabalhador por ampliar suas competências e melhorar seu desempenho em suas tarefas (GEORGE; BRIEF, 1992), participando dos treinamentos oferecidos pela organização e procurando manter-se constantemente atualizado (UEDA, 2012).

Por fim, a virtude cívica remete à atuação do profissional voltada para levantar ameaças e oportunidades para a organização (ALKAHTANI, 2017), contribuir ativamente nas reuniões que discutem as políticas organizacionais, identificar posturas não condizentes no ambiente de trabalho e defender os interesses do negócio (BUKHARI, 2008).

Apesar de ser motivado por diferentes fatores, tais como a postura adotada pela liderança, a consonância entre indivíduo e organização, as características e disposições pessoais dos funcionários, etc. (REGO, 2002; SCHEPERS et al., 2005), o CCO também pode ser encorajado por meio de um processo de avaliação de desempenho capaz de identificá-lo, associado a um sistema de recompensas intrínsecas e extrínsecas que proporcione aos cidadãos organizacionais o devido reconhecimento (IBUKUNOLUWA; ANUOLUWAPO; AGBUDE, 2015; ANDRADE et al., 2017).

O comportamento de cidadania organizacional deve ser valorizado pelos gestores, pois, por meio dos atos espontâneos que gera em prol do negócio, traz diversos benefícios, tais como o aumento da eficiência, da produtividade e da capacidade de a organização se adaptar às mudanças ambientais (PODSAKOFF et al., 2000; FERDUS; KABIR, 2018).

\subsection{SIINDROME DE BURNOUT}

A síndrome de Burnout é uma desordem psíquica que decorre da exposição do sujeito, em seu ambiente laboral, a fatores estressores crônicos (MASLACH; JACKSON, 1981) que o levam ao esgotamento com o passar do tempo (SANTINI; MOLINA NETO, 2005). Apesar de acometer principalmente trabalhadores que lidam diretamente com pessoas em seu trabalho (FREUDENBERGER, 1974), pode manifestar-se em qualquer grupo ocupacional (BRIA et al., 2014).

Esse distúrbio pode desenvolver-se em decorrência de condutas empresariais abusivas e/ou ambientes de trabalho desgastantes (SCHEUER et al., 2016), sobrecarga física e mental advinda das atividades laborais desempenhadas (GUENETTE; SMITH, 2018), não visualização de reconhecimento profissional por parte dos superiores (SHIH et al., 2013), metas inatingíveis (LEE; ASHFORTH, 1996) e falta de oportunidades de crescimento profissional na organização (HAINES III; SABA, 2012).

A síndrome de Burnout possui três dimensões, sendo elas a exaustão emocional, a baixa realização e a despersonalização, cada uma delas responsável por um grupo específico de sintomas (SANTINI, 2004; MASLACH; JACKSON, 1981).

A exaustão emocional refere-se a um estado de fadiga acentuada; o trabalhador sente que não possui mais energia (BACK et al., 2016) ou recursos emocionais para lidar com as diversas demandas provenientes de seu ambiente laboral (HOBFOLL; FREEDY, 1993). Quando a exaustão emocional atinge níveis muito elevados, o indivíduo passa a ter receio de retornar ao trabalho (YU et al., 2015). 
Harrison Bachion Ceribeli, Joice Rayra Vieira Diniz, Raoni de Oliveira Inácio e Israel José dos Santos Felipe

Já a baixa realização pode ser descrita como um sentimento de incompetência que tem origem em uma autoavaliação negativa (FYE; GNILKA; MCLAULIN, 2018). O sujeito sente-se infeliz consigo mesmo e, ao mesmo tempo, insatisfeito com seu desempenho profissional (DALCIN; CARLOTTO, 2018), passando a se autodepreciar e a se sentir um fracasso (KNANI; FOURNIER; BIRON, 2018).

Por fim, a despersonalização, elemento crucial para se diferenciar a síndrome de Burnout e o estresse (ACEVES et al., 2006), caracteriza-se pela objetificação ou coisificação das pessoas. $O$ indivíduo rompe os vínculos afetivos e mantém apenas os racionais em suas relações no trabalho (MASIERO et al., 2018), além de passar a alimentar sentimentos negativos pelos outros, voltando sua conduta para si (MASLACH; SCHAUFELI, 1993).

A síndrome de Burnout está associada a múltiplas consequências deletérias tanto para os indivíduos quanto para as organizações (ACEVES et al., 2006), o que explica sua relevância no universo do trabalho contemporâneo (LEITER; BAKKER; MASLACH, 2014).

Do ponto de vista individual, ocasiona maior irritabilidade, mudanças de humor frequentes (MELAMED et al., 1999), distúrbios do sono (SÖDERSTRÖM et al., 2004), sensação de cansaço persistente (KSIĄŻEK et al., 2011), ansiedade, depressão, baixa autoestima e desmotivação (ACEVES et al., 2006; BANG; REIO, 2017), além de impactar negativamente a saúde e o contexto familiar do trabalhador (GUENETTE; SMITH, 2018). Adicionalmente, leva à dificuldade de concentração, embaraço para lidar com conflitos, baixa tolerância à frustração (NEWELL; MACNEIL, 2010), reduzido envolvimento com o trabalho (LAVIAN, 2012), resistência à cooperação e inflexibilidade (SANTINI, 2004).

Todos esses efeitos negativos da doença acabam prejudicando o desempenho do trabalhador (NIESSEN et al., 2017) e reduzindo sua intenção de permanência na organização (JYOTI; RANI, 2019), resultando em menor eficácia das operações empresariais (GUENETTE; SMITH, 2018). Além disso, ainda levam ao aumento do número de erros e retrabalho (ACEVES et al., 2006), do absenteísmo (IVERSON; OLEKALNS; ERWIN, 1998) e do presenteísmo (BORGES et al., 2016), bem como à diminuição do comprometimento organizacional (GUENETTE; SMITH, 2018) e da cidadania organizacional (LIU; ZHOU; CHE, 2019). Nessa linha, propôs-se a seguinte hipótese de pesquisa:

$H_{1}$ : A síndrome de Burnout pode apresentar uma relação negativa com o comportamento de cidadania organizacional.

\subsection{OPORTUNIDADES DE DESENVOLVIMENTO PROFISSIONAL NAS ORGANIZAÇÕES}

Devido à dinamicidade do mercado, as organizações, para se manterem competitivas ao longo do tempo, precisam apresentar respostas rápidas aos desafios que enfrentam continuamente (RODRIGUEZ; VIVAR; LAZARO, 2017), sendo necessário, para isso, contar com profissionais qualificados (DAVID; COBEANU, 2016). Nesse contexto, no qual o conhecimento tornou-se um dos principais ativos empresariais (CALOF; WRIGHT, 2008), estimular e promover o desenvolvimento humano nas organizações passaram a ser vistas como ações geradoras de diferencial competitivo (SHEEHAN, 2014; SILVA et al., 2015).

Seguindo nessa linha, muitos gestores passaram a investir mais recursos em programas de treinamento e desenvolvimento (T\&D), com o intuito de favorecerem a expansão do portfólio de conhecimentos dos funcionários (ALIYU; NYADZAYO, 2018), contribuindo com o crescimento pessoal, satisfação e motivação deles no trabalho (SUNG; CHOI, 2013) e trazendo benefícios para a organização como o aumento da produtividade e a maior inovação (KNAPIK, 2008; SUNG; CHOI, 2013; SHEEHAN, 2014). 
Subsequentemente, uma vez que os profissionais se tornam mais capacitados em decorrência dos investimentos da organização em T\&D (LACERDA; ABBAD, 2003), passam a ser capazes de assumir novas tarefas (PARKER; MURPHY, 1998; TAMAYO; ABBAD, 2006; CLARKE; HIGGS, 2016), demandando, ao mesmo tempo, novas responsabilidades e também atividades nas quais possam aplicar os conhecimentos adquiridos, o que tem levado diversos gestores a adotarem práticas conhecidas como job enrichment e job enlargement (ALEXANDER, 1975).

A prática de job enrichment (enriquecimento do cargo) consiste no aumento do grau de complexidade das funções desempenhadas pelo profissional e dos desafios que ele enfrenta no trabalho (PAUL; ROBERTSON; HERZBERG, 1969). Por sua vez, a prática de job enlargement (alargamento do cargo) remete à ampliação horizontal dos deveres do funcionário, adicionando-se novas atividades ao trabalho por ele desempenhado (BERDICCHIA; NICOLLI; MASINO, 2016). Em ambos os casos, são proporcionadas oportunidades de aprimoramento ao indivíduo ao mesmo tempo em que lhe são demandados mais esforços, tornando o trabalho menos tedioso e, por conseguinte, uma fonte de maior motivação (BERDICCHIA; NICOLLI; MASINO, 2016).

Além dos aspectos supracitados, cabe chamar a atenção para o novo paradigma de carreira, que aumenta o protagonismo dos indivíduos nas decisões que envolvem sua trajetória profissional, contrapondo a visão tradicional de que a organização é a única responsável pelo crescimento de seus funcionários (REDONDO; SPARROW; HERNÁNDEZLECHUGA, 2019); com isso, o modelo clássico de carreira, linear e gerenciado pela própria organização, tem sido gradualmente substituído pelo modelo de carreira proteana, mais flexível e focada nos objetivos individuais (BRISCOE; FINKELSTEIN, 2009). A questão da carreira é fundamental em um contexto organizacional de estímulo e promoção do desenvolvimento humano, pois proporciona à organização uma ferramenta de retenção dos profissionais mais qualificados, capazes de dar sustentação ao negócio (STOECKICHT; SOARES, 2009).

Quando a organização proporciona oportunidades de desenvolvimento profissional a seus colaboradores, faz com que eles se sintam devidamente reconhecidos (ANTLOGA; TAKAKI, 2016) e seguros (READER et al., 2017), o que tende a elevar seu desempenho (MARKOS; SRIDEVI, 2010), além de sua percepção de justiça, seu comprometimento (FERREIRA et al., 2015) e sua satisfação no trabalho (VALEAU; PAILLÉ, 2019). Por outro lado, a falta de oportunidades de desenvolvimento nas organizações faz com que os profissionais percam o sentimento de pertencimento organizacional (MARTINSA; COSTA; SIQUEIRA, 2016) e apresentem um desempenho laboral abaixo do esperado (NIESSEN et al., 2017), além de maior absenteísmo e rotatividade (WONG; WONG, 2017), exaustão emocional (LEITER; MASLACH, 1988) e estresse ocupacional (DAL PAl et al., 2015). Nessa linha, foram delineadas as seguintes hipóteses de pesquisa:

$\mathrm{H}_{2}$ : As oportunidades de desenvolvimento profissional proporcionadas aos indivíduos nas organizações podem apresentar uma relação positiva com o comportamento de cidadania organizacional.

$\mathrm{H}_{3}$ : As oportunidades de desenvolvimento profissional proporcionadas aos indivíduos nas organizações podem apresentar uma relação negativa com a manifestação da síndrome de Burnout. 
Harrison Bachion Ceribeli, Joice Rayra Vieira Diniz, Raoni de Oliveira Inácio e Israel José dos Santos Felipe

\section{ASPECTOS METODOLÓGICOS}

A presente pesquisa pode ser classificada como descritiva quantitativa, pois, a fim de examinar determinadas características da população estudada, empregaram-se variáveis numéricas para representar os construtos de interesse do estudo e avaliar a relação entre eles, aplicando-se, para isso, técnicas estatísticas de análise.

A população da pesquisa incluiu funcionários de cervejarias multinacionais que atuam no Brasil, englobando todos os níveis hierárquicos. Optou-se pela survey online como método de pesquisa, empregando-se um questionário estruturado para coleta dos dados, o que permitiu que os próprios indivíduos que compõem a população-alvo do estudo fossem utilizados como fontes primárias de informação.

Para elaborar o questionário, adotou-se uma escala de sete pontos do tipo Likert, com o intuito de mensurar a concordância dos respondentes em relação às afirmações apresentadas, sendo nomeados apenas os extremos como "discordo totalmente" e "concordo totalmente". O instrumento foi dividido em cinco seções, sendo a primeira e a segunda reservadas, respectivamente, à coleta de dados demográficos e profissionais dos respondentes, possibilitando a posterior caracterização da amostra. As três seções finais foram destinadas às questões empregadas para mensurar as oportunidades de desenvolvimento profissional, o comportamento de cidadania organizacional e a síndrome de Burnout.

Para avaliar as oportunidades de desenvolvimento profissional concedidas aos profissionais pelas organizações, foram utilizadas 10 questões, sendo três relativas à participação em programas de capacitação, elaboradas a partir de Pilati e Abbad (2005) e Assunção, Carvalho-Freitas e Oliveira (2015), três referentes ao enriquecimento e alargamento do cargo (job enrichment e job enlargement) e quatro relacionadas à carreira, retiradas e adaptadas de Assunção, Carvalho-Freitas e Oliveira (2015). Para avaliar o comportamento de cidadania organizacional, foram retiradas 10 questões da escala ECCO (Escala de Comportamentos de Cidadania Organizacional), proposta por Siqueira (2003). Por fim, para avaliar a manifestação de cada uma das três dimensões da síndrome de Burnout, utilizou-se a versão do Maslach Burnout Inventory (MBI) (MASLACH; JACKSON, 1981) traduzida por Schuster et al. (2016), que conta com 16 itens.

Para compor a amostra da pesquisa, empregou-se a técnica de amostragem não probabilística por conveniência, que permite que as unidades amostrais sejam selecionadas de acordo com o acesso dos pesquisadores e amplitude do estudo, gerando resultados satisfatórios em um menor tempo (OLIVEIRA, 2001). Cabe esclarecer que a escolha desta técnica deveu-se à impossibilidade de obter uma relação contendo todos os funcionários que ocupam cargos de gestão nos níveis estratégico e tático das cervejarias multinacionais que atuam no Brasil, inviabilizando, assim, uma seleção aleatória.

O tamanho amostral foi delimitado adotando-se uma proporção de 10 respondentes para cada item do questionário (HAIR JUNIOR et al., 2009). Considerando que foram utilizadas 36 variáveis, definiu-se que a amostra da pesquisa deveria conter, no mínimo, 360 respondentes.

$\mathrm{Na}$ etapa de coleta de dados, o link de acesso ao questionário elaborado em formato eletrônico na plataforma Google Forms foi enviado pelo chat do Linkedin para o público previamente selecionado. Além disso, o referido link também foi enviado individualmente, utilizando o WhatsApp, para profissionais que preenchiam os critérios pré-definidos.

Para análise dos dados, optou-se pela técnica de Modelagem de Equações Estruturais (MEE), que possibilitou que fossem examinados, de forma simultânea, mais de um construto 
e a relação existente entre eles (HAIR JUNIOR et al., 2009). Testou-se um modelo completo de variáveis latentes, que compreende um modelo de mensuração e um modelo estrutural (BYRNE, 2016). O modelo de mensuração retratou o agrupamento das 36 variáveis observadas do estudo em cinco variáveis latentes, representando os construtos oportunidades de desenvolvimento profissional, comportamento de cidadania organizacional, exaustão emocional, despersonalização e baixa realização pessoal. Por sua vez, o modelo estrutural descreveu a ligação entre as variáveis latentes, o que possibilitou que fossem testadas as hipóteses de pesquisa previamente delineadas.

\section{APRESENTAÇÃO E DISCUSSÃO DOS RESULTADOS}

A amostra da pesquisa foi composta por 384 respondentes, dos quais $77,6 \%$ são do sexo masculino e $22,4 \%$, do feminino. Quanto à faixa etária, 33,6\% possuíam, no momento da coleta de dados, até 29 anos; 46,6\%, entre 30 e 39 anos; e 19,8\%, 40 anos ou mais.

Complementarmente, $34,4 \%$ dos respondentes eram solteiros; $61,2 \%$, casados; e $4,4 \%$ informaram outros estados civis. Além disso, $53,4 \%$ não tinham filhos; $23,2 \%$ tinham um único filho; e $23,4 \%$, dois filhos ou mais. Em relação à escolaridade, 4,1\% possuíam até o ensino médio; $3,1 \%$, curso técnico; $9,4 \%$, ensino superior incompleto; $41,4 \%$, ensino superior completo; e $41,9 \%$, pós-graduação.

Por fim, 9\% dos respondentes trabalhavam há um ano ou menos na organização a qual estavam vinculados no momento em que os dados foram coletados; $10,9 \%$, entre um e dois anos; $23,3 \%$, entre três e cinco anos; 28,9\%, entre seis e 10 anos; e 27,9\% estavam há mais de 10 anos na mesma organização.

Finda a caracterização da amostra, examinou-se a normalidade dos dados coletados, empregando, para isso, a assimetria e a curtose. Adotando um nível de $99 \%$ de confiança, definiu-se como parâmetro aceitável para ambas as medidas um valor dentro do intervalo entre -2,58 e +2,58 (HAIR JÚNIOR et al., 2009), o que permitiu que se confirmasse que todas as variáveis seguiam uma distribuição normal.

Após confirmar que o pressuposto da normalidade não fora violado, testou-se, utilizando o software AMOS, um primeiro modelo completo de variáveis latentes, composto por cinco variáveis latentes representando os construtos exaustão emocional, despersonalização, realização pessoal, oportunidades de desenvolvimento profissional e comportamento de cidadania organizacional, formadas, respectivamente, por seis, quatro, seis, 10 e nove variáveis observadas. Contudo, mesmo após se excluírem todas as variáveis observadas com carga fatorial inferior a 0,7 , os índices de qualidade de ajuste obtidos foram insatisfatórios.

Testou-se, então, um segundo modelo, reduzindo para três o número de variáveis latentes por meio do agrupamento das variáveis observadas relativas às dimensões da síndrome de Burnout (exaustão emocional, despersonalização e realização pessoal) em uma única variável latente, preservando inalteradas as outras duas incorporadas ao modelo anterior (oportunidades de desenvolvimento profissional e comportamento de cidadania organizacional). Novamente, mesmo efetuando a exclusão de todas as variáveis observadas com carga fatorial inferior a 0,7 , não foram obtidos índices de qualidade de ajuste aceitáveis.

Com isso, decidiu-se pela execução de uma Análise Fatorial Exploratória englobando as 36 variáveis originais do estudo, a qual retornou uma solução que foi utilizada como parâmetro para estruturação do terceiro modelo testado, que contou com quatro variáveis latentes, à medida que as dimensões da síndrome de Burnout exaustão emocional e 
Harrison Bachion Ceribeli, Joice Rayra Vieira Diniz, Raoni de Oliveira Inácio e Israel José dos Santos Felipe

despersonalização foram agrupadas em uma única variável latente, enquanto as outras três representaram a realização pessoal, as oportunidades de desenvolvimento profissional e o comportamento de cidadania organizacional.

Assim como foi feito com os dois modelos testados anteriormente, mantiveram-se somente as variáveis observadas que apresentaram cargas fatoriais superiores a 0,7 , visando obter uma estrutura bem definida (HAIR JUNIOR et al., 2009).

Nessa terceira rodada da modelagem, ao contrário do que se observou nas anteriores, foram obtidos valores satisfatórios para os quatro índices de qualidade de ajuste examinados: o qui-quadrado normado foi de 2,417, sendo recomendados valores inferiores a 3,0 (HAIR JUNIOR et al., 2009); o AGFI (adjusted goodness of fit index ou índice de qualidade de ajuste calibrado) foi de 0,82, sendo aceitáveis valores superiores a 0,8 (RHEE; ULEMAN; LEE, 1996; STACCIARINI; PACE, 2017); o GFI (goodness of fit index ou índice de qualidade de ajuste) foi de 0,851, sendo aceitáveis valores próximos ou superiores a 0,9 (SCHUMACKER; LOMAX, 2010); e o RMSEA (root mean square error of approximation ou raiz do erro quadrático médio de aproximação) foi de 0,064 , sendo recomendados valores dentro do intervalo entre 0,05 e 0,08 (HAIR JUNIOR et al., 2009; ARBUCKLE, 2010).

O modelo completo que fora validado com base nos índices de qualidade de ajuste supracitados pode ser visualizado na Figura 1, enquanto a descrição das variáveis observadas que foram agrupadas em cada variável latente foi apresentada no Quadro 1.

Figura 1 - Modelo completo de variáveis latentes elaborado no software AMOS

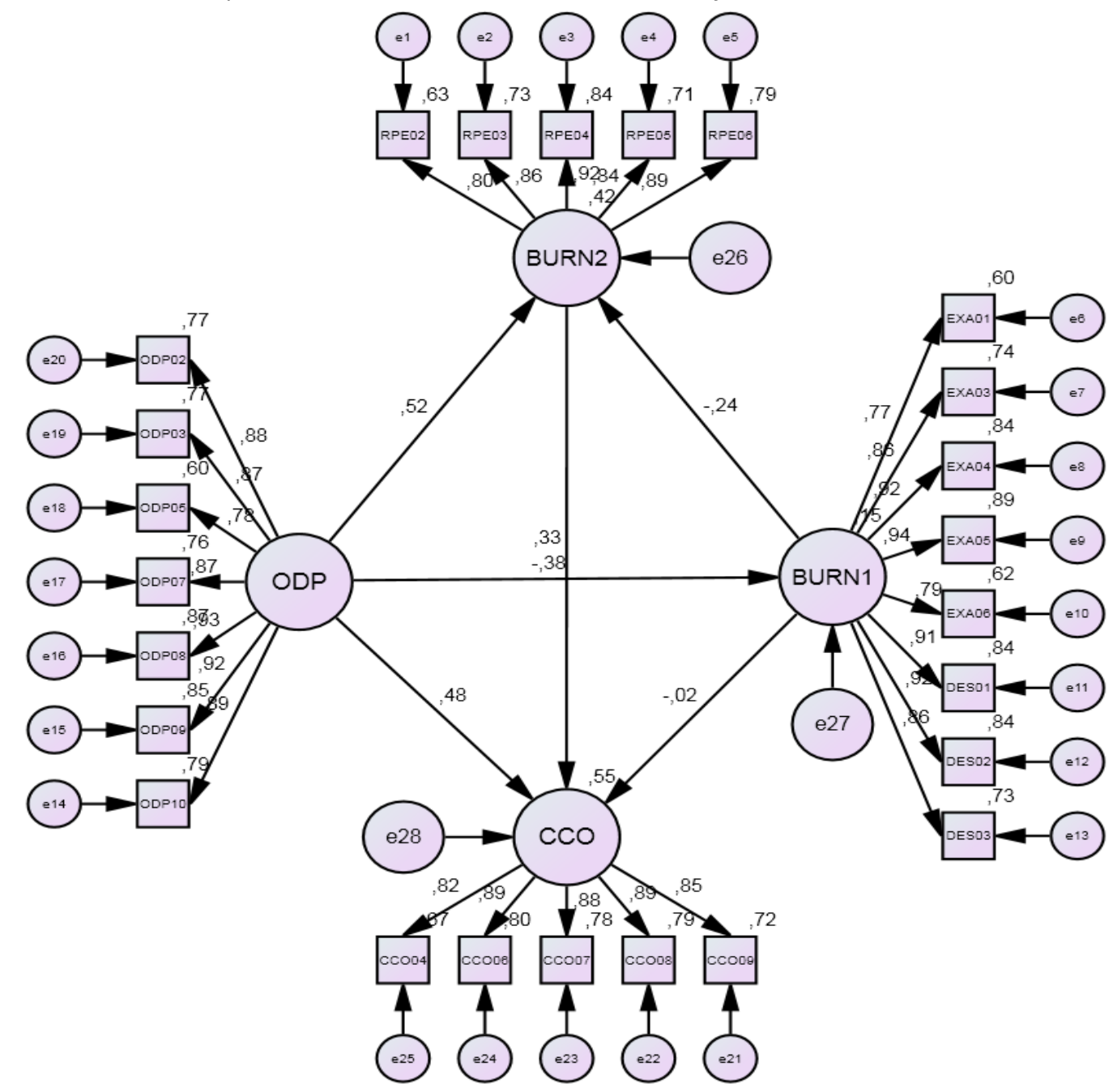

Fonte: elaboração própria (2020). 
Desenvolvimento profissional, Burnout e cidadania organizacional

Quadro 1 - Variáveis observadas incluídas no modelo de mensuração

\begin{tabular}{|c|c|c|}
\hline 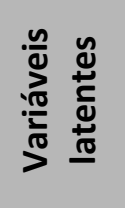 & 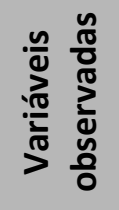 & Descrição \\
\hline \multirow{5}{*}{ BURN2 } & RPE02 & Realizo muitas coisas valiosas no meu trabalho. \\
\hline & RPE03 & Posso efetivamente solucionar problemas que surgem no meu trabalho. \\
\hline & RPE04 & Sinto que estou dando uma contribuição efetiva para essa organização. \\
\hline & RPE05 & Em minha opinião, sou bom no que faço. \\
\hline & RPE06 & $\begin{array}{l}\text { No meu trabalho, sinto-me confiante de que sou eficiente e capaz de fazer com que as } \\
\text { coisas aconteçam. }\end{array}$ \\
\hline \multirow{8}{*}{ BURN1 } & EXA01 & Sinto-me emocionalmente esgotado com o meu trabalho. \\
\hline & EXA03 & $\begin{array}{l}\text { Sinto-me cansado quando me levanto pela manhã e preciso encarar outro dia de } \\
\text { trabalho. }\end{array}$ \\
\hline & EXA04 & Trabalhar todo o dia é realmente motivo de tensão para mim. \\
\hline & EXA05 & Sinto-me acabado por causa do meu trabalho. \\
\hline & EXA06 & Só desejo fazer o meu trabalho sem ser incomodado. \\
\hline & DES01 & Sou menos interessado no meu trabalho desde que assumi essa função. \\
\hline & DESO2 & Sou menos entusiasmado com meu trabalho. \\
\hline & DES03 & Sou mais descrente da contribuição do meu trabalho para algo. \\
\hline \multirow{5}{*}{$\mathrm{CCO}$} & CCOO4 & Ofereço orientação aos colegas menos experientes no trabalho. \\
\hline & CCO06 & Apresento sugestões ao meu chefe para resolver problemas no setor onde trabalho. \\
\hline & CCOO7 & Apresento ao meu chefe ideias novas sobre meu trabalho. \\
\hline & CCO08 & Dou orientação aos colegas que se sentem confusos no trabalho. \\
\hline & CCO09 & $\begin{array}{l}\text { Dou informações positivas sobre essa empresa para as pessoas que me perguntam sobre } \\
\text { ela. }\end{array}$ \\
\hline \multirow{8}{*}{ ODP } & ODP02 & $\begin{array}{l}\text { A empresa na qual trabalho me dá oportunidade de desenvolver novos conhecimentos } \\
\text { e/ou habilidades. }\end{array}$ \\
\hline & ODP03 & Tenho oportunidade de colocar em prática aquilo que aprendo nos treinamentos. \\
\hline & ODP05 & $\begin{array}{l}\text { Ao desenvolver minha capacidade, tenho possibilidade de assumir mais } \\
\text { responsabilidades no trabalho. }\end{array}$ \\
\hline & ODP06 & Sinto-me constantemente desafiado pelas minhas tarefas no trabalho. \\
\hline & ODP07 & $\begin{array}{l}\text { A organização oferece oportunidades de promoção para que eu progrida em minha } \\
\text { carreira. }\end{array}$ \\
\hline & ODP08 & $\begin{array}{l}\text { O desenvolvimento da carreira dos funcionários é uma preocupação da organização } \\
\text { onde trabalho. }\end{array}$ \\
\hline & ODP09 & $\begin{array}{l}\text { O desenvolvimento da carreira é feito de forma compartilhada e conversada entre a } \\
\text { organização e seus funcionários. }\end{array}$ \\
\hline & ODP10 & $\begin{array}{l}\text { Para a organização em que trabalho, as expectativas dos funcionários são consideradas } \\
\text { na gestão de carreira. }\end{array}$ \\
\hline
\end{tabular}

Fonte: elaboração própria (2020).

Depois de confirmar que o modelo estruturado possuía qualidade de ajuste satisfatória, o próximo passo foi a validação do modelo de mensuração, que remete ao

RGO - Revista Gestão Organizacional, Chapecó, v. 14, n. 3, p. 208-228, set./dez. 2021. 
Harrison Bachion Ceribeli, Joice Rayra Vieira Diniz, Raoni de Oliveira Inácio e Israel José dos Santos Felipe

agrupamento das variáveis observadas em variáveis latentes. Para tanto, foram examinados cinco indicadores: $p$-valores, cargas fatoriais, confiabilidade composta (CC), variância média extraída (AVE) e Alfa de Cronbach.

Adotando um nível de confiança de $99 \%$, examinaram-se os p-valores das variáveis observadas. Como somente foram obtidos valores inferiores a 0,001 , confirmou-se que todas elas mantinham uma relação estatisticamente significativa com as variáveis latentes nas quais foram agrupadas. As cargas fatoriais, por sua vez, apresentaram valores superiores a 0,7, conforme já mencionado anteriormente, o que pode ser interpretado como um indicativo da existência de uma estrutura de agrupamentos bem definida (HAIR JUNIOR et al., 2009).

Quanto à variância extraída (VE), confiabilidade composta (CC) e Alfa de Cronbach, que indicam, respectivamente, a precisão dos agrupamentos efetuados, a variância dos itens originais que é explicada pela variável latente e a consistência interna de cada conjunto de variáveis observadas, e que devem apresentar valores superiores a 0,5, 0,7 e 0,7 (HAIR JUNIOR et al., 2009), foram obtidos resultados aceitáveis, conforme pode ser observado na Tabela 1.

Tabela 1 - AVE, CC e Alfa de Cronbach

\begin{tabular}{cccc}
\hline Variáveis Latentes & VE & CC & Alfa de Cronbach \\
\hline BURN2 & 0,7416182 & 0,9942976213 & 0,919 \\
BURN1 & 0,7619626 & 0,9966797048 & 0,944 \\
CCO & 0,7733008 & 0,9953215934 & 0,906 \\
ODP & 0,7711077 & 0,9967787218 & 0,941 \\
\hline
\end{tabular}

Fonte: elaboração própria (2020).

Analisando de maneira concomitante todas as métricas supramencionadas, é possível confirmar a validade do modelo de mensuração delineado e, por conseguinte, das variáveis latentes criadas para representar os construtos examinados na pesquisa. Com isso, procedeuse à análise do modelo estrutural.

Testou-se, primeiramente, a relação entre as oportunidades de desenvolvimento profissional (ODP) e o comportamento de cidadania organizacional (CCO). Foi obtido um pvalor de 0,000 e um coeficiente de caminho igual a 0,481, o que indica que quanto maiores as oportunidades de desenvolvimento que são concedidas pelas indústrias cervejeiras a seus empregados, mais eles tendem a se comportar como cidadãos organizacionais, ratificando, assim, $\mathrm{H}_{2}$.

Em seguida, testou-se a relação entre as oportunidades de desenvolvimento profissional (ODP) e a exaustão emocional e despersonalização (BURN1), sendo obtidos um pvalor de 0,000 e coeficiente de caminho igual a -0,384. Complementarmente, testou-se a relação entre as oportunidades de desenvolvimento profissional (ODP) e a realização pessoal (BURN2), obtendo-se um p-valor de 0,000 e um coeficiente de caminho igual a 0,518. Inferese, portanto, que quanto maiores as oportunidades de desenvolvimento que são concedidas pelas indústrias cervejeiras a seus empregados, menos eles tendem a se sentir exaustos emocionalmente e indiferentes em relação às pessoas com quem trabalham, ao mesmo tempo em que tendem a se sentir mais realizados. Logo, ratifica-se $\mathrm{H}_{3}$.

Subsequentemente, examinou-se a relação entre a exaustão emocional e despersonalização (BURN1) e o comportamento de cidadania organizacional (CCO), para a qual foi encontrado um $p$-valor de 0,721 , o que indica que essas duas dimensões da síndrome de Burnout não exercem uma influência estatisticamente significativa sobre o CCO.

Em contrapartida, para a relação entre a realização pessoal (BURN2) e o comportamento de cidadania organizacional (CCO), obteve-se um p-valor de 0,000 e um 
coeficiente de caminho igual a 0,332, o que indica que, entre os participantes da pesquisa, os indivíduos que se sentem mais realizados tendem a agir como cidadãos organizacionais, enquanto aqueles que apresentam baixo nível de realização pessoal deixam de apresentar comportamentos desse tipo.

Analisando conjuntamente as duas últimas relações examinadas, verifica-se que, no setor cervejeiro, apenas uma das dimensões da síndrome de Burnout - a baixa realização pessoal - pode vir a exercer uma influência direta sobre a disposição dos trabalhadores de se comportarem como cidadãos organizacionais. Contudo, considerando que a exaustão emocional e a despersonalização (BURN1) levam à diminuição da realização pessoal (BURN2) ( $p$-valor de 0,000 e coeficiente de caminho igual a -0,244) e que isto, por sua vez, reduz o comportamento de cidadania organizacional, conclui-se que a síndrome de Burnout pode diminuir a manifestação desse tipo de comportamento no ambiente laboral. Nesse sentido, é possível confirmar $\mathrm{H}_{1}$.

Concluída a apresentação da análise estatística efetuada, torna-se pertinente tecer alguns comentários a respeito dos resultados alcançados, interpretando-os à luz do referencial teórico previamente consultado.

Primeiramente, cabe destacar a constatação de que as organizações que atuam no setor cervejeiro, quando possibilitam que seus funcionários participem de treinamentos e ampliem seu portfólio de conhecimentos, concedendo-lhes, posteriormente, mais responsabilidades e desafios no trabalho, ao mesmo tempo em que lhes oferecem possibilidades de crescimento na carreira, tendem a estimulá-los a se comportarem como verdadeiros cidadãos organizacionais, apresentando atitudes altruístas que incluem uma genuína disposição de auxiliar os colegas (SMITH; ORGAN; NEAR, 1983; WILLIAMS; ANDERSON, 1991) e uma preocupação desinteressada em relação às tarefas em atraso ou de difícil execução (LEAL et al., 2009), além de maior lealdade organizacional, que se manifesta no dia a dia como uma preocupação sincera de preservar a imagem organizacional perante a sociedade (BETTENCOURT; GWINNER; MEUTER, 2001; CORDEIRO; CUNHA, 2018).

Ademais, salienta-se a constatação de que, no âmbito da indústria cervejeira, as oportunidades de desenvolvimento profissional que são proporcionadas aos funcionários, uma vez que diminuem sua exaustão emocional (LEITER; MASLACH, 1988) e a despersonalização, e ampliam o sentimento de autorrealização que experimentam, tendem a reduzir a probabilidade de que eles sejam acometidos pela síndrome de Burnout.

Refletindo a respeito de tais resultados, conclui-se que o indivíduo, à medida que percebe que lhe são concedidas oportunidades para se desenvolver profissionalmente, passa a se sentir mais valorizado (ANTLOGA; TAKAKI, 2016) e seguro (READER et al., 2017), tornando-se mais predisposto a assumir comportamentos favoráveis à organização, adotando uma postura mais colaborativa no ambiente de trabalho e atuando para preservar a imagem organizacional, ao mesmo tempo em que se torna menos sujeito ao esgotamento e distúrbios dele decorrentes, tais como a síndrome de Burnout.

De maneira complementar, debruçando-se sobre a constatação de que a síndrome de Burnout tende a reduzir a cidadania organizacional (LIU; ZHOU; CHE, 2019), conclui-se que o indivíduo, à medida que se sente sem energia (BACK et al., 2016) e recursos emocionais para lidar com as demandas oriundas de seu ambiente laboral (HOBFOLL; FREEDY, 1993), e passa a nutrir sentimentos negativos em relação aos outros (MASLACH; SCHAUFELI, 1993), mantendo somente um vínculo racional em suas relações profissionais (MASIERO et al., 2018), experimenta menor nível de realização pessoal, o que o leva a produzir uma autoavaliação negativa (FYE; GNILKA; MCLAULIN, 2018) e depreciativa (KNANI; FOURNIER; BIRON, 2018) que 
Harrison Bachion Ceribeli, Joice Rayra Vieira Diniz, Raoni de Oliveira Inácio e Israel José dos Santos Felipe

pode diminuir seu envolvimento com o trabalho (LAVIAN, 2012) e ainda ampliar sua resistência a assumir uma postura de efetiva colaboração (SANTINI; 2004).

\section{CONSIDERAÇÕES FINAIS}

Esta pesquisa teve como objetivo analisar, no contexto da indústria cervejeira, a relação entre a síndrome de Burnout, as oportunidades de desenvolvimento profissional que são proporcionadas pelas organizações a seus funcionários e o comportamento de cidadania organizacional.

Examinando os resultados obtidos após as etapas de coleta e análise de dados, constatou-se que, no setor cervejeiro, quando a organização fornece condições para que os funcionários se desenvolvam, eles podem retribuir comportando-se como cidadãos organizacionais, além de estarem menos sujeitos a desenvolverem a síndrome de Burnout.

Adicionalmente, verificou-se que o profissional que se sente emocionalmente exausto e rompe os vínculos afetivos em suas relações de trabalho tende a experimentar menor realização pessoal e, em decorrência disso, deixar de cooperar espontaneamente e defender a imagem da organização frente a eventuais comentários negativos, o que indica que a síndrome de Burnout pode contribuir para inibir o comportamento de cidadania organizacional.

Tais resultados assinalam a importância de propiciar aos funcionários oportunidades de participar de programas de treinamento, visando ampliar o portfólio de competências que possuem; realizar reuniões individuais para auxiliá-los em seu planejamento de carreira; e estruturar um modelo de carreira que consiga contemplar as expectativas que eles nutrem em relação à própria trajetória profissional na organização.

Sob a ótica da Ciência Administrativa, a relevância deste estudo perpassa pela contribuição que fornece para o avanço da compreensão dos fatores que impulsionam ou inibem o comportamento de cidadania organizacional, assim como da relação que existe entre as oportunidades de desenvolvimento profissional concedidas aos trabalhadores e a manifestação da síndrome de Burnout.

Já sob a perspectiva da prática gerencial, este estudo contribui para reforçar a necessidade de se investir no desenvolvimento humano no âmbito das organizações, trazendo benefícios bilaterais na relação entre empregados e empregadores, e para ampliar nos gestores a conscientização de que é necessário prestar atenção aos sinais que possam indicar que determinado funcionário esteja sofrendo devido à síndrome de Burnout, para que ele receba, em tempo hábil, o devido suporte nessa situação.

Apesar de ter alcançado o objetivo traçado, esta pesquisa teve como limitação o fato de ter abarcado um único setor, o das cervejarias, não englobando outros tipos de indústrias e nem outros tipos de negócios, ligados, por exemplo, ao setor de serviços e ao setor de tecnologia, que possuem suas especificidades. Sendo assim, como sugestão para estudos futuros, recomenda-se que o mesmo método e instrumento empregados nesta pesquisa sejam replicados junto a trabalhadores de outros setores, o que irá permitir que os resultados que foram descritos aqui sejam confrontados com os que forem encontrados em outras realidades, aumentando o alcance e a robustez das conclusões obtidas.

Ademais, recomenda-se analisar a influência que cada prática voltada para promover o desenvolvimento dos funcionários nas organizações exerce sobre as diferentes dimensões do construto comportamento de cidadania organizacional, de modo que se obtenha uma visão mais aprofundada a respeito dessa relação que fora aqui descortinada. 


\section{REFERÊNCIAS}

ACEVES, G. A. G.; LÓPEZ, M. A. C.; JIMÉNEZ, S. M.; SERRATOS, F. F.; CAMPOS, J. J. S. Síndrome de burnout. Archivos de Neurociencias, v. 11, n. 4, p. 305-309, 2006.

ALEXANDER, K. O. On work and authority: issues in job enlargement, job enrichment, worker participation and shared authority. The American Journal of Economics and Sociology, v. 34, n. 1, p. 43-54, 1975. DOI: https://doi.org/10.1111/j.1536-7150.1975.tb01157.x.

ALIYU, O. A.; NYADZAYO, M. W. Reducing employee turnover intention: a customer relationship management perspective. Journal of Strategic Marketing, v. 26, n. 3, p. 241257, 2018. DOI: https://doi.org/10.1080/0965254X.2016.1195864.

ALKAHTANI, A. Organizational citizenship behavior (OCB) and rewards. International Business Research, v. 8, n. 4, p. 210-222, 2017. DOI:

http://dx.doi.org/10.5539/ibr.v8n4p210.

ANDRADE, T.; COSTA, V. F.; ESTIVALETE, V. F. B.; LENGLER, L. Comportamento de cidadania organizacional: um olhar à luz dos valores e da satisfação no trabalho. Revista Brasileira de Gestão de Negócios, v. 19, n. 64, p. 236-262, 2017. DOI:

https://doi.org/10.7819/rbgn.v19i64.2899.

ANTLOGA, C. S.; TAKAKI, K. T. O que é qualidade de vida no trabalho? Representações de trabalhadores de um instituto de pesquisa. Trabalho (En) Cena, v. 1, n. 1, p. 132-142, 2016.

ARBUCKLE, J. L. IBM SPSS Amos 19 user's guide. Crawfordville: Amos Development Corporation, 2010.

ASSUNÇÃO, R. V.; CARVALHO-FREITAS, M. N.; OLIVEIRA, M. S. Satisfação no trabalho e oportunidades de desenvolvimento da carreira entre profissionais com deficiência.

Psicologia: Organizações e Trabalho, v. 15, n. 4, p. 340-351, 2015. DOI:

http://dx.doi.org/10.17652/rpot/2015.4.556.

BACK, A. L; STEINHAUSER K. E.; KAMAL, A. H.; JACKSON, V. A. Building resilience for palliative care clinicians: an approach to burnout prevention based on individual skills and workplace factors. Journal of Pain and Symptom Management, v. 52, n. 2, p. 284-291, 2016. DOI: https://doi.org/10.1016/j.jpainsymman.2016.02.002.

BANG, H; REIO, T. G. Examining the role of cynicism in the relationships between burnout and employee behavior. Revista de Psicología del Trabajo y de las Organizaciones, v. 33, n. 3, p. 217-227, 2017. DOI: https://doi.org/10.1016/j.rpto.2017.07.002.

BATEMAN, T. S.; ORGAN, D. W. Job satisfaction and the good soldier: the relationship between affect and employee "citizenship". Academy of Management Journal, v. 26, n. 4, p. 587-595, 1983. DOI: https://doi.org/10.5465/255908. 
Harrison Bachion Ceribeli, Joice Rayra Vieira Diniz, Raoni de Oliveira Inácio e Israel José dos Santos Felipe

BERDICCHIA, D.; NICOLLI, F.; MASINO, G. Job enlargement, job crafting and the moderating role of self-competence. Journal of Managerial Psychology, v. 31, n. 2, p. 318-330, 2016. DOI: https://doi.org/10.1108/JMP-01-2014-0019.

BETTENCOURT, L. A.; GWINNER, K. P.; MEUTER, M. L. A comparison of attitude, personality, and knowledge predictors of service-oriented organizational citizenship behaviors. Journal of Applied Psychology, v. 86, n. 1, p. 29, 2001. DOI: https://doi.org/10.1037/00219010.86.1.29.

BORGES, E. A.; MARGARIDA, A.; QUEIRÓS, C.; BAPTISTA, P.; FELLI, V.; MOSTEIRO, P. Presenteeism among nurses: burnout and engagement as predictors. Journal of Occupational and Environmental Medicine. v. 73, n. 13, p. 201-228, 2016. DOI: http://dx.doi.org/10.1136/oemed-2016-103951.633.

BRIA, M.; SPÂNU, F.; BABAN, A.; DUMITRASCU, D. L. Maslach Burnout Inventory - general survey: factorial validity and invariance among Romanian healthcare professionals. Burnout Research, v. 1, n. 3, p. 103-111, 2014. DOI: https://doi.org/10.1016/j.burn.2014.09.001.

BRISCOE, J. P.; FINKELSTEIN L. M. The "new career" and organizational commitment: do boundaryless and protean attitudes make a difference? Career Development International, v. 14, n. 3, p. 242-260, 2009. DOI: https://doi.org/10.1108/13620430910966424.

BUKHARI, Z. U. Key antecedents of organizational citizenship behavior (OCB) in the banking sector of Pakistan. International Journal of Business and Management, v. 3, n. 12, p. 106115, 2008. DOI: https://doi.org/10.5539/ijbm.v3n12p106.

BYRNE, B. M. Structural Equation Modeling with Amos: basic concepts, applications, and programming. 3. ed. New York: Routledge, 2016.

CALOF, J. L.; WRIGHT, S. Competitive intelligence: a practitioner, academic and interdisciplinary perspective. European Journal of Marketing, v. 42, n.8, p.717-730, 2008. DOI: https://doi.org/10.1108/03090560810877114.

CLARKE, N.; HIGGS, M. How strategic focus relates to the delivery of leadership training and development. Human Resource Management, v. 55, n. 4, p. 541-565, 2016. DOI: https://doi.org/10.1002/hrm.21683.

CORDEIRO, J. P.; CUNHA, P. Gestão de conflitos, comportamentos de comprometimento e de cidadania organizacional em contexto educativo: contributos para a formulação de um modelo conceitual. European Journal of Applied Business Management, v. 4, n. 3, p. 46-66, 2018.

DAL PAI, D.; LAUTERT, L.; SOUZA, S. B. C.; MARZIALE, M. H. P.; TAVARES, J. P. Violência, burnout e transtornos psíquicos menores no trabalho hospitalar. Revista da Escola de Enfermagem da USP, v. 49, n. 3, p. 457-464, 2015. DOI: https://doi.org/10.1590/S0080623420150000300014. 
DALCIN, L.; CARLOTTO, M. S. Evaluation of the effect of an intervention for Burnout Syndrome in teachers. School and Educational Psychology, v. 22, n. 1, p. 141-150, 2018. DOI: https://doi.org/10.1590/2175-35392018013718.

DAVID, O. A.; COBEANU, O. Evidence-based training in cognitive-behavioural coaching: can personal development bring less distress and better performance? British Journal of Guidance \& Counselling, v. 44, n. 1, p. 12-25, 2016. DOI: https://doi.org/10.1080/03069885.2014.1002384.

FERDUS, Z.; KABIR, T. Effect of job satisfaction and organizational commitment on OCB: study on private banks in Bangladesh. World Journal of Social Sciences, v. 8, n. 2, p. 57-69, 2018.

FERREIRA, M. C. O.; SILVA, J. G.; BIZARRIAS, F. S.; CARVALHO, J. B.; SOUZA, F. M. S.; FRANÇA, M. H.; GONÇALVES, M. S. Políticas e práticas de gestão de pessoas e suas relações com o absenteísmo na área de produção: desafios ao desenvolvimento sustentável. Labor e Engenho, v. 9, n. 3, p. 87-97, 2015. DOI: https://doi.org/10.20396/lobore.v9i3.8634453.

FREUDENBERGER, H. J. Staff burn-out. Journal of Social Issues, v. 30, n. 1, p. 159-165, 1974. DOI: https://doi.org/10.1111/j.1540-4560.1974.tb00706.x.

FYE, H. J.; GNILKA, P. B.; MCLAULIN, S. E. Perfectionism and school counselors: differences in stress, coping, and burnout. Journal of Counseling \& Development, v. 96, n. 4, p. 349-360, 2018. DOI: https://doi.org/10.1002/jcad.12218.

GEORGE, J. M.; BRIEF, A. P. Feeling good-doing well: a conceptual analysis of the mood at work-organizational spontaneity relationship. Psychology Bulletin, v. 112, n. 2, p. 310-329, 1992. DOI: https://doi.org/10.1037/0033-2909.112.2.310.

GEORGE, J. M.; JONES, G. R. Organizational spontaneity in context. Human Performance, n. 10, v. 2, p. 153-170, 1997. DOI: https://doi.org/10.1207/s15327043hup1002_6.

GONTIJO, F. E. K.; GESSENER, E. Logística de distribuição e estratégias de cervejarias em Santa Catarina. XI Congresso Nacional de Excelência em Gestão e II Inovarse, 2015, Rio de Janeiro. Anais... Rio de Janeiro, 2015.

GRAHAM, J. W. An essay on organizational citizenship behavior. Employee Responsibilities and Rights Journal, v. 4, n. 4, p. 249-270, 1991. DOI: https://doi.org/10.1007/BF01385031.

GUENETTE, J. P.; SMITH, S. E. Burnout: job resources and job demands associated with low personal accomplishment in united states radiology residents. Academic Radiology, v. 25, n. 6, p. 739-743, 2018. DOI: https://doi.org/10.1016/j.acra.2017.12.002.

HAINES III, V. Y; SABA, T. Challenges to professional identities and emotional exhaustion. Career Development International, v. 17, n. 2, p.120-136, 2012. DOI:

https://doi.org/10.1108/13620431211225313. 
Harrison Bachion Ceribeli, Joice Rayra Vieira Diniz, Raoni de Oliveira Inácio e Israel José dos Santos Felipe

HAIR JUNIOR, J. F.; BLACK, W. C; BABIN, B. J; ANDERSON, R. E; TATHAM, R. L. Análise multivariada de dados. Porto Alegre: Bookman, 2009.

HOBFOLL, S. E.; FREEDY, J. Conservation of resources: a general stress theory applied to burnout. In: SCHAUFELI; W. B.; MASLACH, C.; MAREK, T. (Eds.). Professional burnout: recent developments in theory and research. Philadelphia: Taylor \& Francis, 1993. p. 115-133.

IBUKUNOLUWA, O. E.; ANUOLUWAPO, A. G.; AGBUDE, G. A. Benefits of organizational citizenship behaviours for individual employees. Covenant International Journal of Psychology, v. 1, n. 1, p. 50-69, 2015.

IVERSON, R. D.; OLEKALNS, M.; ERWIN, P. J. Affectivity, organizational stressors, and absenteeism: a causal model of burnout and its consequences. Journal of Vocational Behavior, v. 52, n. 1, p. 1-23, 1998. DOI: https://doi.org/10.1006/jvbe.1996.1556.

JYOTI, J.; RANI, A. Role of burnout and mentoring between high performance work system and intention to leave: moderated mediation model. Journal of Business Research, v. 98, p. 166-176, 2019. DOI: https://doi.org/10.1016/j.jbusres.2018.12.068.

KNANI, M.; FOURNIER, P. S.; BIRON, C. Psychosocial risks, burnout and intention to quit following the introduction of new software at work. Work, v. 60, n. 1, p. 95-104, 2018. DOI: https://doi.org/10.3233/WOR-182714.

KNAPIK, J. Gestão de pessoas e talentos. São Paulo: Editora Ibpex, 2008.

KSIAZŻEK, I.; STEFANIAK, T.J.; STADNYK, M.; KSIĄŻEK, J. Burnout syndrome in surgical oncology and general surgery nurses: a cross-sectional study. European Journal of Oncology Nursing, v. 15, n. 4, p. 347-350, 2011. DOI: https://doi.org/10.1016/j.ejon.2010.09.002.

LACERDA, É. R. M.; ABBAD, G. Impacto do treinamento no trabalho: investigando variáveis motivacionais e organizacionais como suas preditoras. RAC - Revista de Administração Contemporânea, v. 7, n. 4, p. 77-96, 2003. DOI: https://doi.org/10.1590/S141565552003000400005.

LAVIAN, R. H. The impact of organizational climate on burnout among homeroom teachers and special education teachers (full classes/individual pupils) in mainstream schools.

Teachers and Teaching, v. 18, n. 2, p. 233-247, 2012. DOI:

https://doi.org/10.1080/13540602.2012.632272.

LEAL, S.; BERNARDINO, V.; FARIA, J.; VIVAS, C. A relação entre o perfil motivacional e os comportamentos de cidadania organizacional: um estudo empírico. Jornadas Hispano-Lusas de Gestión Científica, v. 14, n. 19, p. 1-13, 2009.

LEE, R. T; ASHFORTH, B. E. A meta-analytic examination of the correlates of the three dimensions of job Burnout. Journal of Applied Psychology, v. 81, n. 2, p. 123-133, 1996. DOI: https://doi.org/10.1037/0021-9010.81.2.123.

LEITER, M. P.; BAKKER; A. B. MASLACH, C. The contemporary context of job burnout. In: LEITER, M. P.; BAKKER; A. B. MASLACH, C. Burnout at work: a psychology perspective. New York: Psychology Press, 2014. p. 1-9. 
LEITER, M. P.; MASLACH, C. The impact of interpersonal environment on burnout and organizational commitment. Journal of Organizational Behavior, v. 9, n.4, p. 297-308, 1988. DOI: https://doi.org/10.1002/job.4030090402.

LIMBERGER, S. C.; MARTINS, C. A. A. Vantagens competitivas do oligopólio cervejeiro e a permanência de microcervejarias no Brasil. Revista Formação, v. 25, n. 44, p.53-76, 2018. DOI: https://doi.org/10.33081/formacao.v25i44.5151.

LIU, W.; ZHOU, Z. E.; CHE, X. X. Effect of Workplace incivility on OCB through Burnout: the moderating role of affective commitment. Journal of Business and Psychology, v. 34, p. 657669, 2019. DOI: https://doi.org/10.1007/s10869-018-9591-4.

MARKOS, S.; SRIDEVI, M. S. Employee engagement: the key to improving performance. International Journal of Business and Management, v. 5, n. 12, p. 89-96, 2010. DOI: https://doi.org/10.5539/ijbm.v5n12p89.

MARTINSA, V.; COSTA, L. V.; SIQUEIRA, M. M. M. O impacto do comprometimento afetivo e do engajamento no trabalho sobre os comportamentos de cidadania organizacional. RACEF Revista de Administração, Contabilidade e Economia da FUNDACE, v. 6, n. 2, p. 1-13, 2016. DOI: http://dx.doi.org/10.13059/racef.v6i2.327.

MASIERO, M. C. I.; RUSSO, S.; MAZZOCCO, K.; PRAVETTONI, G. Psycho-cognitive predictors of burnout in healthcare professionals working in emergency departments. Journal of Clinical Nursing, v. 13, n.14, p. 2691-2698, 2018. DOI: https://doi.org/10.1111/jocn.14376.

MASLACH, C.; JACKSON, S. E. The measurement of experienced Burnout. Journal of Occupational Behavior, v. 2, n. 14, p. 99-113, 1981. DOI:

https://doi.org/10.1002/job.4030020205.

MASLACH, C. S.; SCHAUFELI, W. B. Historical and conceptual development of burnout. In: SCHAUFELI, W. B.; MASLACH, C. S.; MAREK, T. (Eds.). Professional burnout: recent developments in theory and research. Philadelphia: Taylor \& Francis, 1993. p. 1-16.

MELAMED, S.; UGARTEN, U.; SHIROM, A.; KAHANA, L.; LERMAN, Y.; FROOM, P. Chronic Burnout, somatic arousal and elevated salivary cortisol levels. Journal of Psychosomatic Research, v. 46, n. 6, p. 591-598, 1999. DOI: https://doi.org/10.1016/S00223999(99)00007-0.

MENEZES, I. G.; AGUIAR, C. V. N.; BASTOS, A. V. B. Comprometimento organizacional: questões que cercam sua natureza e os seus limites conceituais. Psicologia em Revista, $v$. 22, n. 3, p. 768-789, 2016. DOI: http://dx.doi.org/DOI-10.5752/P.1678-

9523.2016V22N3P768.

MIAO, C.; HUMPHREY, R. H.; QIAN, S. Are the emotionally intelligent good citizens or counterproductive? a meta-analysis of emotional intelligence and its relationships with organizational citizenship behavior and counterproductive work behavior. Personality and Individual Differences, v. 116, p. 144-156, 2017. DOI:

https://doi.org/10.1016/j.paid.2017.04.015. 
Harrison Bachion Ceribeli, Joice Rayra Vieira Diniz, Raoni de Oliveira Inácio e Israel José dos Santos Felipe

MOTOWIDLO, S. J.; BORMAN, W. C.; SCHMIT, M. J. A theory of individual differences in task and contextual performance. Human Performance, v. 10, n. 2, p. 71-83, 1997. DOI: https://doi.org/10.1207/s15327043hup1002_1.

NEWELL, J, M.; MACNEIL, G. A. Professional burnout, vicarious trauma, secondary traumatic stress, and compassion fatigue. Best Practices in Mental Health, v. 6, n. 2, p. 57-68, 2010.

NIESSEN, C.; MÄDER, I.; STRIDE, C. B.; JIMMIESON, N. Thriving when exhausted: the role of perceived transformational leadership. Journal of Vocational Behavior, v. 103, p. 41-51, 2017. DOI: https://doi.org/10.1016/j.jvb.2017.07.012.

OLIVEIRA T. M. V. Amostragem não probabilística: adequação de situações para uso e limitações de amostras por conveniência, julgamento e quotas. Revista Administração On Line, v. 2, n. 3, p. 1-15, 2001.

PARKER, S. K.; MURPHY, K. R. Enhancing role breadth self-efficacy: the roles of job enrichment and other organizational interventions. Journal of Applied Psychology, v. 83, n. 6, p. 835-852, 1998. DOI: https://doi.org/10.1037/0021-9010.83.6.835.

PAUL, W. J.; ROBERTSON, K. B.; HERZBERG, F. Job enrichment pays off. Harvard Business Review, v. 47, n. 2, p. 61-78, 1969.

PILATI, R.; ABBAD, G. Análise fatorial confirmatória da escala de impacto do treinamento no trabalho. Psicologia: Teoria e Pesquisa, v. 21, n. 1, p. 43-51, 2005. DOI: https://doi.org/10.1590/S0102-37722005000100007.

PODSAKOFF, P. M.; MACKENZIE, S. B.; PAINE, J. B.; BACHRACH, D. G. Organizational citizenship behaviours: a critical review of the theoretical and empirical literature and suggestions for future research. Journal of Management, v. 26, n. 3, p. 513-563, 2000. DOI: https://doi.org/10.1016/S0149-2063(00)00047-7.

PORTO, J. B.; TAMAYO, Á. Valores organizacionais e civismo nas organizações. RAC- Revista de Administração Contemporânea, v. 9, n. 1, p. 35-52, 2005. DOI: https://doi.org/10.1590/S1415-65552005000100003.

READER, T. W., MEARNS, K., LOPES, C.; KUHA, J. Organizational support for the workforce and employee safety citizenship behaviors: a social exchange relationship. Human relations, v. 70, n. 3, p. 362-385, 2017. DOI: https://doi.org/10.1177/0018726716655863.

REDONDO, R.; SPARROW, P.; HERNÁNDEZ-LECHUGA, G. The effect of protean careers on talent retention: examining the relationship between protean career orientation, organizational commitment, job satisfaction and intention to quit for talented workers. The International Journal of Human Resource Management, 2019. DOI: https://doi.org/10.1080/09585192.2019.1579247.

REGO, A. Climas éticos e comportamentos de cidadania organizacional. Revista Administração de Empresas, v. 42, n. 1, p. 1-14, 2002. DOI: http://dx.doi.org/10.1590/S0034-75902002000100006. 
RHEE, E; ULEMAN, J. S; LEE, H. K. Variations in collectivism and individualism by ingroup and culture: Confirmatory Factor Analysis. Journal of Personality and Social Psychology, v. 71, n. 5, p. 1037-1054, 1996. DOI: https://doi.org/10.1037/0022-3514.71.5.1037.

RODRIGUEZ, A. S.; VIVAR, R. M.; LAZARO, J. M. Gestión por competencias laborales en el contexto del proceso de cambios políticos y económicos en Cuba. Innovar, v. 27, n. 66, p. 169-184, 2017. DOI: https://doi.org/10.15446/innovar.v27n66.66811.

SANTINI, J. Síndrome do esgotamento profissional: revisão bibliográfica. Movimento, v. 10, n. 1, p. 183-209, 2004.

SANTINI, J.; MOLINA NETO, V. A síndrome do esgotamento profissional em professores de educação física: um estudo na rede municipal de ensino de Porto Alegre. Revista Brasileira de Educação Física e Esporte, v. 19, n. 3, p. 209-222, 2005. DOI:

https://doi.org/10.1590/S1807-55092005000300004.

SCHEPERS, C.; GIETER, S.; PEPERMANS, R.; BOIS, C. D.; CAERS, R.; JEGERS, M. How are employees of the nonprofit sector motivated? A research need. Nonprofit Management and Leadership, v. 16, n. 2, p. 191-208, 2005. DOI: https://doi.org/10.1002/nml.100.

SCHEUER, M. L.; BURTON, J. P.; BARBER, L. K.; FINKELSTEIN, L. M.; PARKER, C. P. Linking abusive supervision to engagement and Burnout. Organization Management Journal, v. 13, n. 3, 138-147, 2016. DOI: https://doi.org/10.1080/15416518.2016.1214063.

SCHUMACKER, R. E.; LOMAX, R. G. A beginner's guide to structural equation modeling. 3. ed. New York: Routledge, 2010.

SCHUSTER, M. S. DIAS, V. V.; BATTISTELLA, L. F.; GROHMANN, M. Z. MBI-GS scale validation: a general survey investigation on employees' health perception. REGE - Revista de Gestão, v. 22, n. 3, p. 403-416, 2016. DOI: https://doi.org/10.5700/rege569.

SHEEHAN, M. Investment in training and development in times of uncertainty. Advances in Developing Human Resources, v. 16, n. 1, p. 13-33, 2014. DOI:

https://doi.org/10.1177/1523422313508924.

SHIH, S.; JIANG, J. J; KLEIN, G; WANG, E. Job burnout of the information technology worker: work exhaustion, depersonalization, and personal accomplishment. Information \& Management, v. 50, p. 582-589, 2013. DOI: https://doi.org/10.1016/j.im.2013.08.003.

SINDICERV - SINDICATO NACIONAL DA INDÚSTRIA DA CERVEJA. O setor em números. Disponível em: https://sindicerv.com.br/o-setor-em-numeros/. Acesso em: 23 jun. 2019.

SILVA, J. L. L. DA.; SOARES, R. S.; COSTA, F. S.; RAMOS, D. S.; LIMA, F. B.; TEIXEIRA, L. R. Fatores psicossociais e prevalência da síndrome de burnout em trabalhadores de enfermagem em unidades de terapia intensiva. Revista Brasileira de Terapia Intensiva, v. 27, n. 2, p. 125-133, 2015. DOI: https://doi.org/10.5935/0103-507X.20150023.

SIQUEIRA, M. M. M. Proposição e análise de um modelo para proposição e análise de um modelo para comportamentos de cidadania organizacional comportamentos de cidadania organizacional. RAC - Revista de Administração Contemporânea, v. 7, p. 165-184, 2003. DOI: https://doi.org/10.1590/S1415-65552003000500009. 
Harrison Bachion Ceribeli, Joice Rayra Vieira Diniz, Raoni de Oliveira Inácio e Israel José dos Santos Felipe

SMITH, A.; ORGAN, D.; NEAR, J. Organizational citizenship behavior: its nature and antecedents. Journal of Applied Psychology, v. 68, n. 4, p. 653-663, 1983. DOI: https://doi.org/10.1037/0021-9010.68.4.653.

SOARES, E. F.; SIENA, T. M. Comportamento de cidadania organizacional: estudo em organizações no ramo do comércio. Diálogos: Economia e Sociedade, v. 2, n. 1, p. 34-44, 2018.

SÖDERSTRÖM, M.; EKSTEDT, M.; AKERSTEDT, T.; NILSSON, J.; AXELSSON, J. Sleep and sleepiness in young individuals with high burnout scores. Sleep, v. 27, n. 7, p. 1369-1377, 2004. DOI: https://doi.org/10.1093/sleep/27.7.1369.

STACCIARINI, T. S. G.; PACE, A. E. Análise fatorial confirmatória da escala: appraisal of self care agency scale - revised. Revista Latino-Americana de Enfermagem, v. 25, p. 1-9, 2017. DOI: https://doi.org/10.1590/1518-8345.1378.2856.

STOECKICHT, I. P.; SOARES, C. A. P. O capital intelectual, os capitais do conhecimento e a inovação: a importância da gestão estratégica do capital intelectual no desenvolvimento da capacidade de inovação em empresas brasileiras. XXII Simpósio de Administração da Produção, Logística e Operações Internacionais, 2009, São Paulo. Anais... São Paulo: FGV, 2009.

SUNG, S. Y.; CHOI, J. N. Do organizations spend wisely on employees? Effects of training and development investments on learning and innovation in organizations. Journal of organizational behavior, v. 35, p. 393-412, 2013. DOI: https://doi.org/10.1002/job.1897.

TAMAYO, N.; ABBAD, G. S. Autoconceito profissional e suporte à transferência e impacto do treinamento no trabalho. Revista de Administração Contemporânea, v. 10, n. 3, p. 9-28, 2006. DOI: https://doi.org/10.1590/S1415-65552006000300002.

UEDA, Y. Self-development as a form of organizational citizenship behavior: examining the effects of job satisfaction and task characteristics. Keizai, v. 43, n. 1, p. 115-133, 2012.

VALEAU, P. J.; PAILLÉ, P. The management of professional employees: linking progressive HRM practices, cognitive orientations and organizational citizenship behavior. The International Journal of Human Resource Management, v. 30, n. 19, p. 2705-2731, 2019. DOI: https://doi.org/10.1080/09585192.2017.1332671.

WILLIAMS, L. J.; ANDERSON, S. E. Job satisfaction and organizational commitment as predictors of organizational citizenship and in-role behaviors. Journal of Management, v. 17, n. 3, p. 601-617, 1991. DOI: https://doi.org/10.1177/014920639101700305.

WONG, Y. W.; WONG, Y. T. The effects of perceived organisational support and affective commitment on turnover intention: a test of two competing models. Journal of Chinese Human Resource Management, v. 8, n.1, p.2-21, 2017. DOI: https://doi.org/10.1108/JCHRM-01-2017-0001.

YU, X.; WANG, P.; ZHAI, X.; DAI, H.; YANG, Q. The effect of work stress on job burnout among teachers: the mediating role of self-efficacy. Social Indicators Research, v. 122, n. 3, p. 701708, 2015. DOI: https://doi.org/10.1007/s11205-014-0716-5. 